\title{
RESEARCH
}

Open Access

\section{Cerebrospinal fluid A beta 1-40 peptides increase in Alzheimer's disease and are highly correlated with phospho-tau in control individuals}

Sylvain Lehmann ${ }^{1 *}$ D, Julien Dumurgier ${ }^{2}$, Xavier Ayrignac ${ }^{3}$, Cecilia Marelli $^{3}$, Daniel Alcolea ${ }^{4}$, Juan Fortea Ormaechea ${ }^{4}$, Eric Thouvenot ${ }^{5}$, Constance Delaby ${ }^{1}$, Christophe Hirtz' ${ }^{1}$, Jérôme Vialaret ${ }^{1}$, Nelly Ginestet ${ }^{1}$, Elodie Bouaziz-Amar ${ }^{2}$, Jean-Louis Laplanche ${ }^{2}$, Pierre Labauge ${ }^{3}$, Claire Paquet ${ }^{2}$, Alberto Lleo ${ }^{4}$, Audrey Gabelle ${ }^{6}$ and for the Alzheimer's Disease Neuroimaging Initiative (ADNI)

\begin{abstract}
Background: Amyloid pathology, which is one of the characteristics of Alzheimer's disease (AD), results from altered metabolism of the beta-amyloid (AB) peptide in terms of synthesis, clearance, or aggregation. A decrease in cerebrospinal fluid (CSF) level $A \beta 1-42$ is evident in $A D$, and the CSF ratio $A \beta 42 / A \beta 40$ has recently been identified as one of the most reliable diagnostic biomarkers of amyloid pathology. Variations in inter-individual levels of A $1-40$ in the CSF have been observed in the past, but their origins remain unclear. In addition, the variation of AB40 in the context of AD studied in several studies has yielded conflicting results.

Methods: Here, we analyzed the levels of A $\beta 1-40$ using multicenter data obtained on 2466 samples from six different cohorts in which CSF was collected under standardized protocols, centrifugation, and storage conditions. Tau and p-tau (181) concentrations were measured using commercially available in vitro diagnostic immunoassays. Concentrations of CSF A $\beta 1-42$ and A $31-40$ were measured by ELISA, XMAP technology, chemiluminescence immunoassay (CLIA), and mass spectrometry. Statistical analyses were calculated for parametric and non-parametric comparisons, linear regression, correlation, and odds ratios. The statistical tests were adjusted for the effects of covariates (age, in particular).

(Continued on next page)
\end{abstract}

\footnotetext{
* Correspondence: s-lehmann@chu-montpellier.fr

Data used in preparation of this article were obtained from the Alzheimer's Disease Neuroimaging Initiative (ADNI) database (adni.loni.usc.edu). As such, the investigators within the ADNI contributed to the design and implementation of ADNI and/or provided data but did not participate in the analysis or writing of this report. A complete listing of ADNI investigators can be found at: http://adni.loni.usc.edu/wp-content/uploads/how_to_apply/ ADNI_Acknowledgement_List.pdf

'Univ Montpellier, CHU Montpellier (LBPC-PPC), INSERM (IRMB, INM), Montpellier, France

Full list of author information is available at the end of the article
}

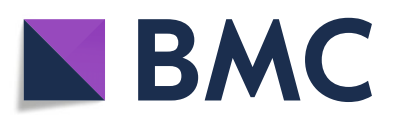

(- The Author(s). 2020 Open Access This article is licensed under a Creative Commons Attribution 4.0 International License, which permits use, sharing, adaptation, distribution and reproduction in any medium or format, as long as you give appropriate credit to the original author(s) and the source, provide a link to the Creative Commons licence, and indicate if changes were made. The images or other third party material in this article are included in the article's Creative Commons licence, unless indicated otherwise in a credit line to the material. If material is not included in the article's Creative Commons licence and your intended use is not permitted by statutory regulation or exceeds the permitted use, you will need to obtain permission directly from the copyright holder. To view a copy of this licence, visit http://creativecommons.org/licenses/by/4.0/. The Creative Commons Public Domain Dedication waiver (http://creativecommons.org/publicdomain/zero/1.0/) applies to the data made available in this article, unless otherwise stated in a credit line to the data. 
(Continued from previous page)

Results: Regardless of the analysis method used and the cohorts, a slight but significant age-independent increase in the levels of $A \beta 40$ in CSF was observed in AD. We also found a strong positive correlation between the levels of A 1-40 and p-tau (181) in CSF, particularly in control patients.

Conclusions: These results indicate that an increase in the baseline level of amyloid peptides, which are associated with an increase in p-tau (181), may be a biological characteristic and possibly a risk factor for AD. Further studies will be needed to establish a causal link between increased baseline levels of Aß40 and the development of the disease.

Keywords: Alzheimer's disease, Amyloid peptides, Tau proteins, Biomarkers, Cerebrospinal fluid (CSF)

\section{Background}

Alzheimer's disease (AD) neuropathological brain lesions consist of aggregates of hyper-phosphorylated tau proteins, which have also been called neurofibrillary tangles (NFTs), and extracellular deposits of amyloid precursor protein (APP) derived amyloid-beta (A $\beta$ ) peptides, which are known as amyloid plaques. Much research has been focusing recently on the molecular mechanisms underlying these pathological events as it has become essential to develop preventive and therapeutic strategies for AD. For a long time, the main explanation for the pathogenesis of $\mathrm{AD}$ was that amyloidogenesis was the primum movens of the affection, which led to the concept of the amyloid cascade [1]. According to this picture of the disease, the alteration of APP metabolism (increasing amyloid production, decreasing clearance rates), the aggregation of $A \beta$ peptides, and the formation of amyloid plaques might result in microglial and astrocyte activation, local inflammatory responses, oxidative stress, and eventually the hyper-phosphorylation of tau proteins and secondarily the formation of NFTs [2].

The idea that amyloid peptides contribute importantly to the etiology of $\mathrm{AD}$ is supported by cases of $\mathrm{AD}$ who carry presenilins ( 1 or 2 ) or APP mutations [3]. These gene mutations trigger the overproduction of $A \beta$ peptides or the preferential production of $A \beta 42$, which is the most amyloidogenic of all the peptides. An APP gene dose effect triggering $\mathrm{AD}$ development, as occurs in Down syndrome [4] and in gene duplication processes [5], is a further/an additional potential factor contributing to amyloid pathogenesis. Other genetic factors have been described, such as apolipoprotein E4 allele, in particular [6].

Studies on cerebrospinal fluid (CSF) biomarkers in AD have greatly improved our understanding of the pathophysiology of this disease. The production of amyloid peptides following the neuronal processing of APP has been involved in the response to physiological challenge with neurotrophic, anti-microbial, tumor suppression, or synaptic function regulation activities [7]. Regarding tau proteins which are associated to microtubules, their physiological secretion by neuronal cells is a recent discovery which physiological relevance and benefit are still matter of debate [8]. A decrease in CSF A $\beta 42$ is especially indicative of an amyloidogenic process, while an increase in tau proteins (total tau and its phosphorylated form p-tau (181)) is known to be associated with axonal loss and tau pathology in $\mathrm{AD}[9,10]$. Tests on these two biomarkers are being included nowadays in the international clinical research guidelines [11, 12], and many centers [11-15], and ourselves [13-15] have integrated them into daily clinical practice. Importantly, these biochemical CSF measurements are concordant with the results of the PET imaging approaches which were initially developed to determine the brain amyloid load [16], and now also serve to measure tau accumulation [17]. These data are in line with hypotheses put forward by Jack et al. [18] about the chronology of the evolution of biomarkers during the pathophysiological process, and the relevance of amyloid markers in particular at a very early stage, probably as early as 10 to 15 years before the onset of clinical symptoms.

Under non-pathological conditions, $A \beta 40$ is highly correlated with $A \beta 42$ [19]. The computation of the ratio A $\beta 42 / 40$ is now being used in routine clinical practice on AD patients in some centers [20-22]. This is a useful approach for reducing pre-analytical A $\beta 42$ biases [23-25] and improving the diagnostic performances of CSF biomarkers [26], especially in discordant cases [27]. This ratio can also be used to account for inter-individual amyloid variations in the baseline CSF level [28]. Low CSF A $\beta 40$ levels might also be indicative of frontotemporal dementia (FTD) [29, 30], cerebral amyloid angiopathy (CAA) [31], HIV [32], multiple sclerosis [33], or normal pressure hydrocephalus (NPH) [34].

In their meta-analysis of AD biomarkers, Olsson et al. [35] observed the existence of a negligible difference in CSF A $\beta 40$ between AD and control patients. Most of the 32 studies considered had a limited number of patients included in each group (the median number of subjects per group was less than 30 , and the maximum number of subjects was 137 and 328 in the $\mathrm{AD}$ and non-AD groups, respectively). The focus of these studies was also quite different, looking at the diagnostic interest of $\mathrm{A} \beta 42 / 40$ in $\mathrm{AD}$ and of $\mathrm{A} \beta$ peptides in other neurodegenerative diseases, or being more interested in pathophysiological mechanisms. 
The rationale for this study is therefore based on several elements: first, to resolve the controversy conflicting studies showing or not an increase in $A \beta 40$ in $\mathrm{AD}$; second, to provide explanations for the very good diagnostic performance of the $A \beta 42 / 40$ ratio calculation; and finally, in the context of the pathophysiology of the disease, to provide elements linking, in sporadic $\mathrm{AD}$, the level of expression of amyloid peptides and the disease. We revisited the issue of the $A \beta 40$ levels using large series of multicenter data. The results show the occurrence of a significant age-independent increase in CSF A 340 in AD. Another noteworthy finding was the existence of a strong positive correlation between CSF A $\beta 40$ and the p-tau (181) concentration, even in patients without Alzheimer's disease (NAD). These findings suggest that the baseline amyloid peptide level may constitute a risk factor contributing to sporadic $\mathrm{AD}$, which is associated with p-tau (181) production.

\section{Methods}

\section{Study design and subjects}

Patients with cognitive impairments were recruited and followed at the Montpellier and Paris Memory Resources Center (CMRR). The Montpellier participants were subdivided into two cohorts which were recruited during different periods: Montpellier 1 (Mtp-1) (recruited from July 2015 to May 2017) and Montpellier 2 (Mtp-2) (recruited from September 2009 to June 2015). These two periods corresponded to the use of different ELISA kits (SupTable 1). The cohort Mtp-1 consisted of 400 patients (126 AD, 274 NAD), and the cohort Mtp-2 consisted of 504 patients (220 AD, 284 NAD). The Paris cohort consisted of 624 patients (299 AD, 325 NAD) from the Centre de Neurologie Cognitive, Groupe Hospitalier Lariboisière Fernand-Widal (recruited from March 2012 to May 2017). The Barcelona SPIN (Sant Pau Initiative on Neurodegeneration) cohort (79 AD, 148 NAD) consisted of patients who had undergone lumbar puncture for CSF AD biomarkers at the Sant Pau Memory Unit [36, 37] (recruited from May 2009 to December 2017). All the patients underwent a thorough clinical examination including biological lab tests, neuropsychological assessments, and brain imaging. The same diagnostic procedure [27] and AD diagnostic criteria [11] were used at all the clinical centers which participated. We included in the $\mathrm{AD}$ group patients which, in the absence of substantial concomitant cerebrovascular disease, meet the $\mathrm{AD}$ core clinical criteria and are considered as probable $\mathrm{AD}$ or possible $\mathrm{AD}$ with biomarker evidence of AD (CSF amyloid, tau, FDG-PET, or structural MRI) [11]. Although CSF amyloid biomarkers were included in the diagnostic criteria for $\mathrm{AD}$, we observed that between 2 and $7 \%$ of $\mathrm{AD}$ cases, depending on the cohort, were not based on evidence of amyloid biomarkers but rather on other elements such as MRI. The possible AD category without amyloid or tau biomarker evidence of $\mathrm{AD}$ corresponds to the mild cognitive impairment (MCI) in the absence of core features of synucleinopathies, frontotemporal dementia (FTD), primary progressive aphasia, or evidence for another concurrent, active neurological disease, or a non-neurological medical comorbidity or use of medication that could have a substantial effect on cognition. Subjective cognitive impairment (SCI) corresponds to a situation where a patient reports an alteration of their cognitive functions, including memory, but this cannot be documented by clinical, neuropsychological, imaging, or biological tests.

The NAD diagnosis included SCI, CAA, NPH, FTD based on relevant criteria [38], dementia with Lewy bodies (based on the McKeith criteria [39]), corticobasal degeneration (based on the criteria defined by Boeve et al. [40]), progressive supranuclear palsy, amyotrophic lateral sclerosis, and vascular dementia (based on the usual consensus diagnostic criteria). All the patients at each clinical center gave their written informed consent to participating in clinical research on CSF biomarkers, which was approved by the respective Ethics Committees. The committee responsible in Montpellier was the regional Ethics Committee of the Montpellier University Hospital and Montpellier CSF-Neurobank \#DC-2008417 at the certified NFS 96-900 CHU resource center BB-0033-00031, www.biobanques.eu. Authorization to handle personal data was granted by the French Data Protection Authority (CNIL) under the number $1709743 \mathrm{v0.}$

Two sets of data originating from the analysis of CSF samples at the Alzheimer's Disease Neuroimaging Initiative (ADNI) database (www.loni.ucla.edu/ADNI) were used after the agreement of the scientific committee. ADNI UPEN-RESULTS, UPEN-ELYCYS $(n=311)$, and MS UPENNMSMSABETA $(n=400)$ data were also used. In the ADNI cohorts, which included many patients with mild cognitive impairments (MCI), we had to rely on the biological PLM (Paris-Lille-Montpellier) scale [41] to define populations with a low $(\mathrm{ADNI}(-))$ and high $(\mathrm{ADNI}(+))$ prevalence of $\mathrm{AD}$. This scale combines the concentration of the three CSF biomarkers $[A \beta 42$, tau, p-tau (181)] into a probability scale for AD. The score ranges from 0 to 3 based on the number of abnormal CSF biomarkers. ADNI(-) population corresponded to PLM scale of 0 or 1 with less than $25 \%$ of $A D$, while $\mathrm{ADNI}(+)$ corresponded to PLM scale of 2 and 3 with more than $75 \%$ of AD. Importantly, the PLM score used was not based on the $A \beta 40$ values so as to prevent circular reasoning. This way of stratifying patients in the ADNI cohort represents anyway a limitation of our study.

\section{CSF samples and assays}

CSF was collected using standard conditions of collection, centrifugation, and storage [42, 43]. CSF tau and p-tau 
(181) concentrations were measured using the standardized commercially available INNOTEST $_{R}$ sandwich ELISA, Luminex ${ }^{\circ} \mathrm{xMAP}$ technology $(\mathrm{x}=$ analyte, MAP $=$ Multi-Analyte Profiling) assays in line with the manufacturer's instructions (Fujirebio-Europe). The consistency of the p-tau (181) detection using the ELISA assays is ensured by its comparison with the mass spectrometry detection performed in this fluid [44]. In the Mtp-1 cohort, CSF A $\beta 1-42$ and $A \beta 1-40$ (denoted here by $A \beta 42$ and $A \beta 40$ ) were measured with Euroimmun kits (EQ-6511-9601 (A $\beta 1-40)$; EQ-6521-9601 (A $\beta 1-42))$. In the Mtp-2 and Paris cohorts, CSF $A \beta 42$ and $A \beta 40$ were measured using INNOTEST $_{R}$ sandwich ELISA from IBL and Fujirebio, respectively, as recommended by the manufacturer. Roche Elecsys automated chemiluminescence immunoassay (CLIA) and mass spectrometry were used on the ADNI cohorts to measure CSF $A \beta 1-42$ and $A \beta 1-40$ as previously described $[45,46]$. Detection limits of these kits are compatible with CSF clinical ranges. Average concentration of analytes may differ between kits in relation with standard value assignments by the vendors in the absence of certified reference materials.

The pre-analytical procedure was standardized [43] but differed, depending on the type of collection tubes used [36, 47]. This explains the differences observed between cohorts in the mean $A \beta 40$ and $A \beta 42$ values measured with the same detection kit (SupTable 1). The quality of the results was ensured by using validated standard operating procedures and internal quality controls (QCs). The QC coefficient of variation obtained on the CSF analytes in each batch and between batches ranged consistently below $15 \%$. In addition, external QC procedures were used to confirm the quality/accuracy of the results [42]. In the case of the ADNI cohorts, CSF samples were deep frozen after the lumbar puncture without performing any centrifugation or aliquoting, and shipped to the UPENN ADNI Biomarker Laboratory in Philadelphia on dry ice, where they were thawed, aliquoted, and re-frozen.

\section{Statistical analysis}

Statistical analyses were computed with the MedCalc software program (18.11.3). Data tested for normality were expressed either as means \pm SDs or as median 25th/75th percentile, and differences between groups were taken to be significant in the Student $t$ tests or the non-parametric Mann-Whitney $U$ test at $P<0.05$. Linear regression was computed between continuous biomarkers, and the corresponding Pearson correlation coefficients and statistical significance have been specified in the tables. For meta-analysis of the study, we used the Hedges $g$ statistic as a formulation for the standardized mean difference (SMD) under the fixed effects model. The SMD Hedges $g$ is the difference between the two means divided by the pooled standard deviation. When indicated, statistical tests were adjusted to account for the effects of covariates (age, in particular). Odds ratios corresponded to the presence of $\mathrm{AD}$ in the various percentile groups, based on the distribution of $A \beta 40$. The $95 \%$ confidence odds ratio intervals were computed along with the $z$-statistics and the associated $P$ values.

\section{Results}

\section{CSF $A \beta 42$ and $A \beta 40$ in $A D$ and NAD populations}

CSF data on 2466 samples originating from six different cohorts were included in the present study. The AD and NAD populations were defined based on clinical criteria in the Montpellier 1 (Mtp-1), Montpellier 2 (Mtp-2), Paris, and Barcelona cohorts. For the ADNI cohort, we relied on the PLM score to distinguish $\mathrm{ADNI}(+)$ and ADNI(-) population. Differences were observed in terms of age and CSF biomarker profiles in the overall population, as well as in each clinical cohort (Table 1). As was to be expected, AD patients obtained lower MMSE scores and showed higher CSF tau and p-tau (181) levels than NAD patients/participants. A significant decrease in the CSF A 42 concentrations was observed in the AD population (Fig. 1a), regardless of the cohort tested. Noteworthy differences were also observed in the A $\beta 40$ levels between all the cohorts (Fig. 1b): the values recorded in the AD population were significantly higher than in the NAD group regardless of analytical method, the sex, or the age as covariate. We then conducted a meta-analysis study including the four independent cohorts using the Hedges $g$ statistic as a formulation for the standardized mean difference (SMD) under the fixed effects model (Fig. 2). The overall SMD with 95\% CI is at 0.5 , which is greater than 0.2 , the significance level. The difference between $\mathrm{AD}$ and NAD population for A $\beta 40$ was however limited with an important overlap resulting in AUCs lower than 0.8 (Sup Figure 2). The stratification of the ADNI population using the PLM scale (combining $A \beta 42$, tau, and p-tau (181)) limits the interpretation of the $A \beta 40$ results of this cohort in our study. We have nevertheless observed that the $\operatorname{ADNI}(+)$ group, which by definition has less $A \beta 42$, did indeed have more $A \beta 40$ (Fig. 1c). The physiological correlation between CSF $A \beta 40$ and $A \beta 42$ [19] is therefore not well preserved in AD.

When we stratified and combined the clinical cohort populations into $\mathrm{AD}, \mathrm{MCI}, \mathrm{FTD}$, Control (SCI), and other neurological diseases (Other) groups, the difference between $\mathrm{AD}$ and the other clinical groups was eventually confirmed (Fig. 3a). We also performed a more in-depth comparison, stratified by clinical groups. Importantly, the Paris and SPIN-Barcelona cohorts are composed of only a small number of pathologies whereas the Montpellier cohorts have a wide range of diagnoses corresponding to the Memory consultations (see the "Methods" section). As 


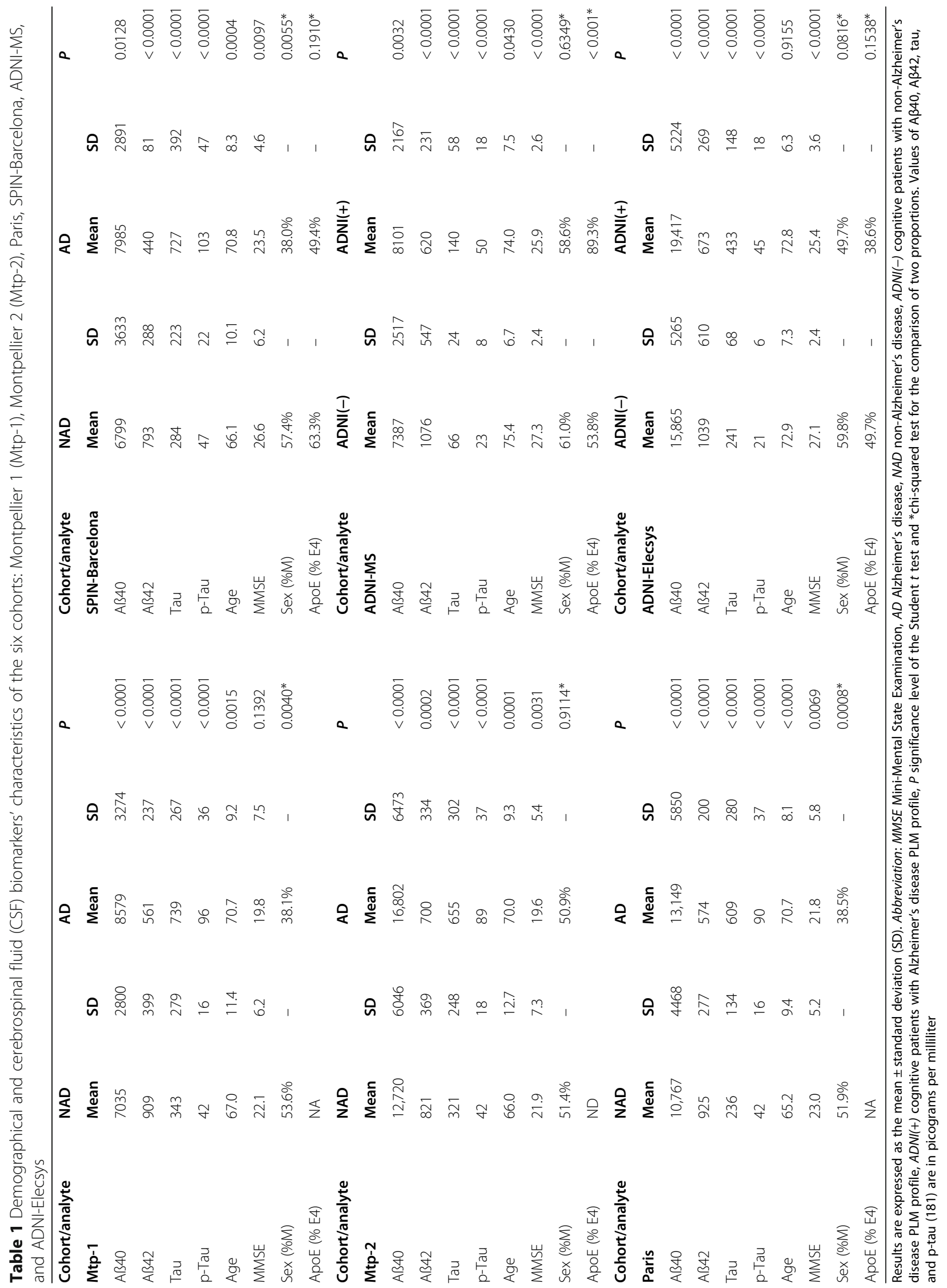




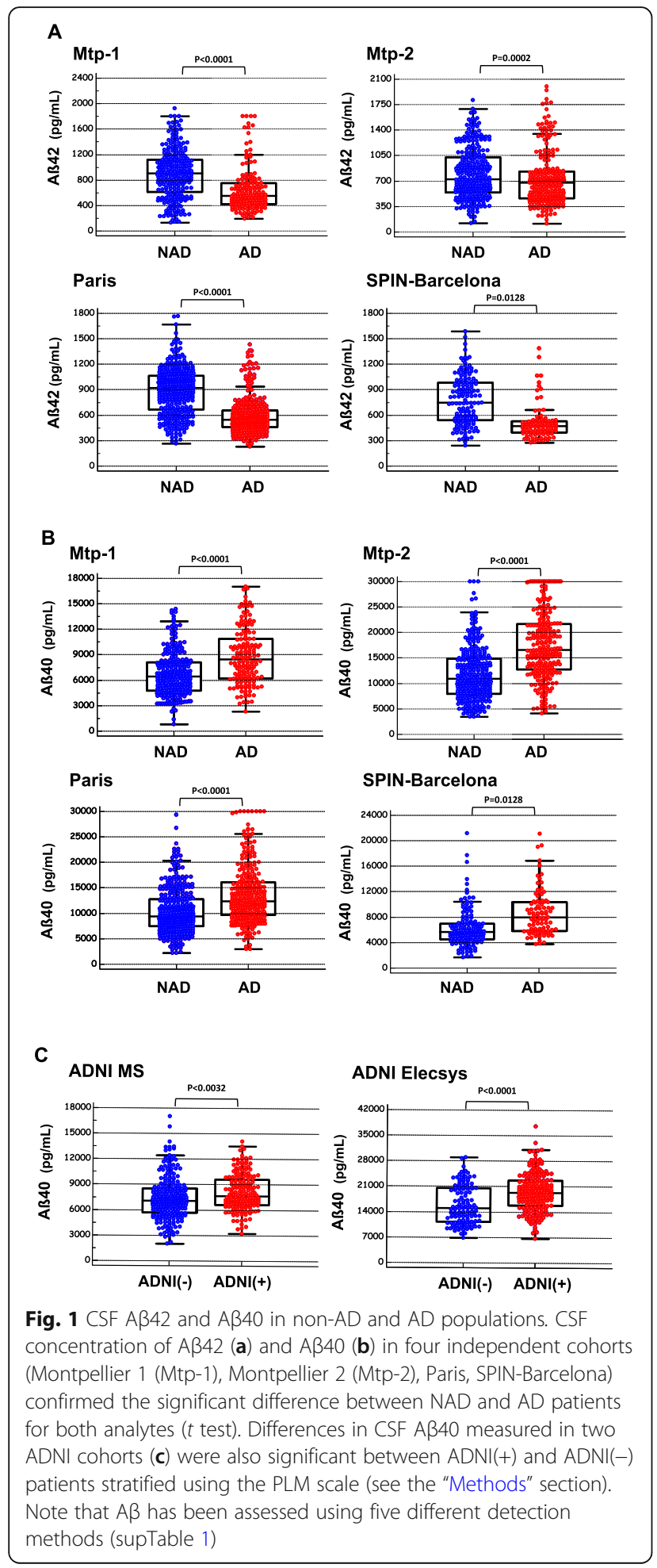

illustrated in the Sup Figure 3, we observed that $\mathrm{AD}$ patients have significantly higher levels of $A ß 40$ than most other diagnostic groups, with the exception of MCI (in two out of three cohorts, there was no statistical difference between $\mathrm{AD}$ and MCI patients). However, this result is not unexpected since the MCI group is itself heterogeneous, with some patients progressing to AD in subsequent years. As expected from previous studies [29, 30, 48], lowered Aß40 values for CAA and FTD were found. This is even more pronounced for NPH, as expected [34]. Based on this stratification, we can conclude that the increase in $A \beta 40$ in the $A D$ population is not a bias related to the presence of certain diagnoses possibly present in the NAD group.

The influence of $A P O E$ status, which was available in the case of 983 samples (524 NAD with $36.6 \% \mathrm{E} 4+; 459$ $\mathrm{AD}$ with $58.4 \% \mathrm{E} 4+)$, was also assessed with respect to the $\mathrm{A} \beta$ levels (SupFigure 1A-F). As previously reported $[49,50]$, the presence of ApoE4 was significantly associated with lower $A \beta 42$ levels, as well as lower $A \beta 40$ levels. The difference in $A \beta 40$ values between NAD and $\mathrm{AD}$ patients was also observed in both ApoE4 positive and negative populations (SupFigure 1G-I).

To investigate more closely the relationship between $A \beta 40$ and $A D$ diagnosis, the total population was sorted into four percentile classes based on the value of this biomarker in the CSF $(<25$ th, 25th-50th, 50th-75th, and $>75$ th percentiles). The percentage of AD patients in each cohort clearly increased along with the $A \beta 40$ percentiles (SupTable2). To account for the differences in $\mathrm{AD}$ prevalence between the cohorts, the odds ratios for $\mathrm{AD}$ were plotted in the case of increasing $\mathrm{A} \beta 40$ percentile classes, and a significant increase ranging from 0.4 to 1.8 was observed (Fig. 3b). To establish whether the difference in age observed between NAD and AD patients (SupTable 1) might be a significant determinant here, the age distribution between percentile classes was also plotted (Fig. 3c). A significant difference in age distribution was observed only between the 50th/ 75th and the $>75$ th A $\beta 40$ percentile classes (Fig. 3c). Age cannot therefore account for the association between A $\beta 40$ levels and AD prevalence. Nevertheless, further statistical tests were adjusted using age as covariate.

\section{Correlations between $A \beta 40$ and the other CSF biomarkers} The correlation between $A \beta 40$ and the other CSF biomarkers was computed in global, NAD, and AD populations, for each cohort, and in the overall population (Table 2). As was to be expected [19], a correlation was found to exist between $A \beta 40$ and $A \beta 42$, especially in the NAD group. A $\beta 40$ was also correlated with the tau levels, and it was striking that the highest correlation coefficients were obtained with p-tau (181) rather than with $\mathrm{t}$-tau, especially in the Mtp-1 cohort (a significant difference was observed between the correlation coefficients at $P=0.02$ ). The correlation was clearly visible when the mean-centered $p$-tau (181) values were plotted in the various $A \beta 40$ percentile classes (Fig. $3 d$ ), showing significant differences between classes. This correlation 


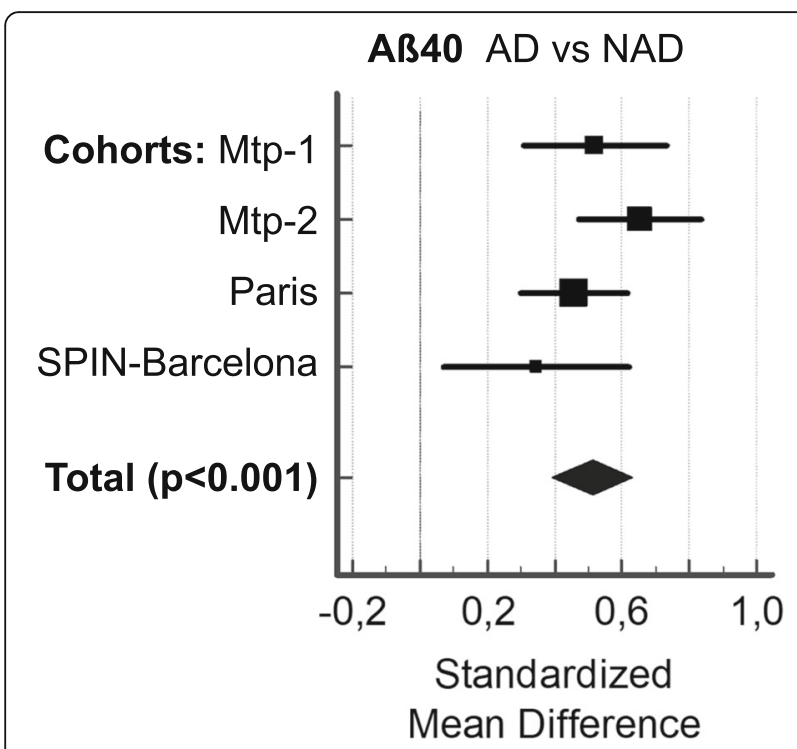

Fig. 2 Meta-analysis including the four independent cohorts. We used with to compare the $A \beta 40$ means between $A D$ and NAD populations the Hedges $g$ statistic as a formulation for the standardized mean difference (SMD) under the fixed effects model. The SMD Hedges $g$ is the difference between the two means divided by the pooled standard deviation. The plot has marker sizes relative to study weight. The results of the different studies, with $95 \% \mathrm{Cl}$, and the overall standardized mean difference with $95 \% \mathrm{Cl}$ are shown. Cohen's rule of thumb for interpretation of the SMD statistic is that a value of 0.2 indicates a small effect, a value of 0.5 indicates a medium effect, and a value of 0.8 or larger indicates a large effect

had to be put in perspective with the fact that both analytes increased in AD with the patients' age (SupTable 1), which justifies the adjustments made for age in our statistical analysis. We illustrated graphically the correlation between p-tau (181) and A $\beta 40$ in the all NAD and the AD populations (Fig. 4a, b). We noticed that p-tau (181) values were higher and more widely distributed in the $\mathrm{AD}$ population with a regression slope that was higher than in the NAD population. To document further the relationship between $A \beta 40$ and p-tau (181) outside the context of $\mathrm{AD}$, the correlation was tested in a series of clinically defined patients with multiple sclerosis [51] and FTD [52] (Fig. 4c, d). The corresponding correlation coefficients were both significantly higher in these groups than in the $\mathrm{AD}$ population $(P<0.001)$.

\section{Discussion}

The decrease in $\mathrm{A} \beta 42$ observed in the CSF of $\mathrm{AD}$ patients has attracted considerable attention in clinical and research communities. This decrease is attributable to the accumulation of $A \beta 42$ in the brain parenchyma, along with a decrease in the rates of CSF clearance and an increase in the production of oligomeric/multimeric forms. Since determining the CSF A $342 / A \beta 40$ ratio provides a useful means of improving AD diagnosis [26], many groups are now also measuring $A \beta 40$ in their patients. A meta-analysis was however not conclusive regarding its differential levels in $\mathrm{AD}$ [35]. Looking back in detail at various studies, A $\beta 40$ either was lower or showed no significant changes [21, 29, 30,53], or apparently increased in $\mathrm{AD}$ in comparison with other forms of dementia [54, 55]. In a recent report, the increase in $A \beta 40$ was clearly identified as one of the reasons for the good performances of the $A \beta 42 / A \beta 40$ ratio as an index [20], while another study on PET amyloid findings also established that CSF A $\beta 40$ increased in the PIB+ population [56]. These discrepancies might be linked to differences in cohort composition, since the A $\beta 40$ levels may be affected by various pathological conditions [29-31]. The stage of $\mathrm{AD}$, corresponding to various levels of cerebral atrophy probably reducing amyloid production [18], may also account for differences between studies. This is coherent with a recent study confirming the increase of $A \beta 40$ in prodromal AD [57]. Differences in the precision of the analytical methods used, combined with the size of the cohorts, might also explain why only a small, nonsignificant difference between $\mathrm{AD}$ and NAD patients has been observed in some cases.

Nevertheless, the present study on CSF A $\beta 40$ which confirms it increased in AD can be considered a "surprise." The fact that this result has not been clearly identified previously is due to several factors, the first being that since the differences between populations are small, statistical significance requires a larger number of samples and measurement methods that are as accurate as possible. Using large cohorts, we were able to confirm the existence of an age- and ApoE-independent increase in CSF A $\beta 40$ in AD compared with other diagnostic consisting mostly of controls and patients with other neurodegenerative diseases and dementia. This observation is valid in different analytical contexts despite differences in the threshold or range for biomarker measurement. Our finding provides additional explanations for the very good diagnostic performance of the $A \beta 42 / 40$ ratio calculation [20]. Interestingly, Janelidze et al. [58] observed that some $A \beta 42$ assays were partly quenched by $A \beta 40$. An increase of $A \beta 40$ in $A D$ as suggested by our study may therefore also indirectly contribute to the diagnostic performance of $A \beta 42$. It is worth mentioning that in the blood, where the amyloid peptide $42 / 40$ ratio could well indicate the presence of brain amyloidosis $[59,60]$, it has been established that high $A \beta 40$ levels are associated with greater mortality rate in the elderly [61]. The fact that the CSF and blood amyloid levels are poorly correlated, however, makes it difficult at this stage to extend the present conclusions to this fluid.

The overlap in the CSF $A \beta 40$ values between the AD and NAD populations is worth noting, and the area under the receiver operating characteristic curve (AUC) for $\mathrm{AD}$ diagnosis was under 0.8 in all the cohorts tested 


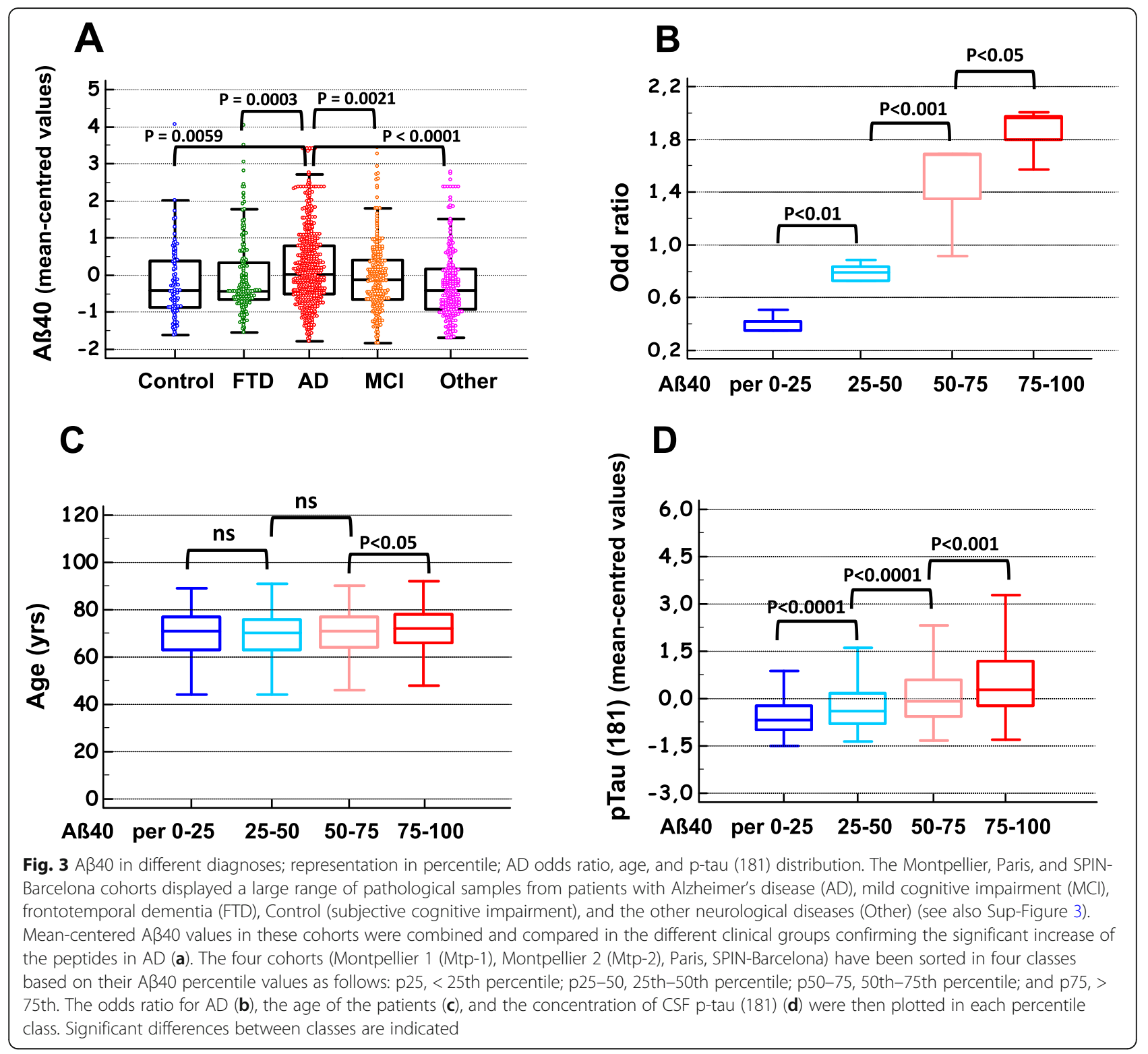

Table 2 Age-adjusted Pearson's correlation between A 440 and A 442, tau, or p-tau (181) values in the six cohorts (Montpellier 1 (Mtp-1), Montpellier 2 (Mtp-2), Paris, SPIN-Barcelona, ADNI-MS, ADNI-Elecsys), in the overall population using mean-centered values to account for level differences between analytical methods

\begin{tabular}{|c|c|c|c|c|c|c|c|c|c|}
\hline \multirow{2}{*}{$\begin{array}{l}A B 40 \\
\text { correlation }\end{array}$} & \multicolumn{3}{|l|}{ All } & \multicolumn{3}{|c|}{ NAD or ADNI(-) } & \multicolumn{3}{|c|}{ AD or $A D N I(+)$} \\
\hline & p-Tau & Tau & Aß42 & p-Tau & Tau & $A ß 42$ & p-Tau & Tau & $A ß 42$ \\
\hline Mtp-1 & 0.501 & 0.377 & 0.520 & 0.557 & $0.235^{*}$ & 0.764 & 0.504 & 0.443 & 0.613 \\
\hline Mtp-2 & 0.514 & 0.443 & 0.407 & 0.430 & 0.244 & 0.533 & 0.304 & $0.213^{*}$ & 0.469 \\
\hline Paris & 0.419 & 0.36 & 0.188 & 0.421 & $0.145^{*}$ & 0.571 & 0.288 & 0.245 & 0.247 \\
\hline SPIN-Barcelona & 0.468 & 0.396 & 0.161 & 0.483 & 0.348 & 0.419 & 0.355 & $0.256^{*}$ & $0.308^{*}$ \\
\hline ADNI-MS & 0.254 & 0.413 & 0.505 & 0.257 & 0.496 & 0.705 & $0.223^{*}$ & 0.470 & 0.574 \\
\hline ADNI-Elecsys & 0.536 & 0.567 & 0.449 & 0.487 & 0.624 & 0.782 & 0.502 & 0.496 & 0.580 \\
\hline Overall & 0.445 & 0.418 & 0.368 & 0.455 & 0.318 & 0.615 & 0.339 & 0.318 & 0.426 \\
\hline
\end{tabular}

Computation has been done in the All population and in the $\mathrm{NAD}, \mathrm{AD}, \mathrm{ADNI}(-)$, and $\mathrm{ADNI}(+)$ groups. Correlation coefficient statistical value $P<0.001$ for all but $* P<0.01$ 


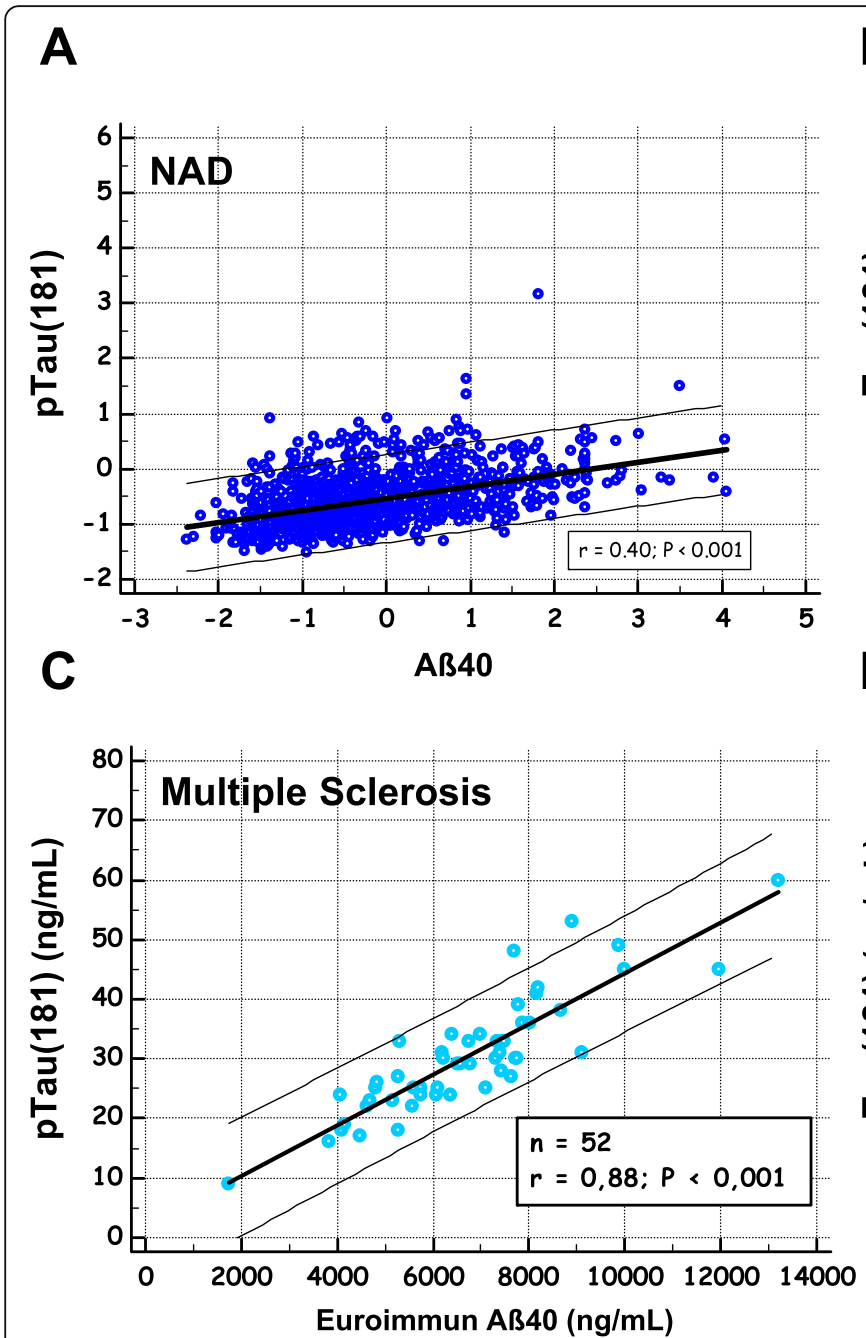

B
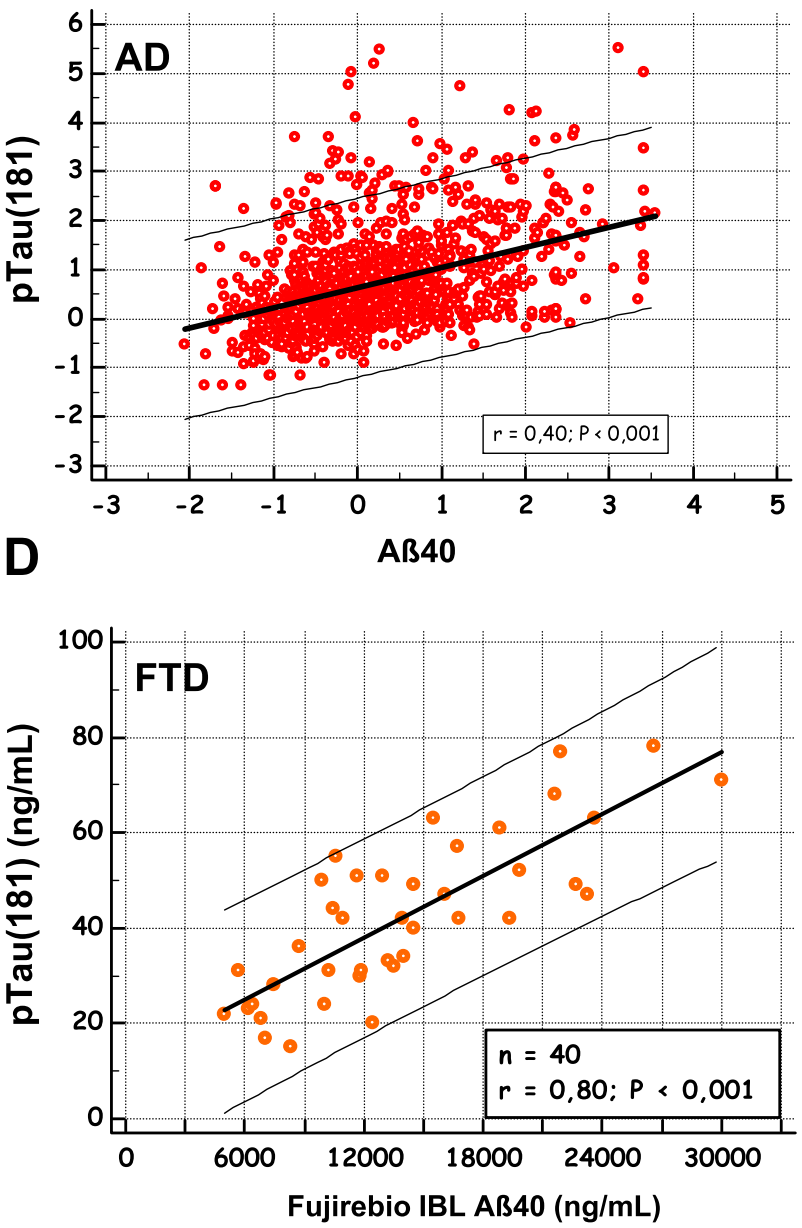

Fig. 4 Correlation between $A \beta 40$ and p-tau (181) in different clinical populations. To illustrate the correlation between A 440 and p-tau (181) (Table 2), the mean-centered concentrations of the two analytes in the total study population were plotted in NAD (a) and AD populations (b). A 40 and p-tau (181) concentrations were also plotted in a selection of multiple sclerosis (c) and FTD patients (d)

(SupFigure2). CSF A $\beta 40$ cannot therefore be used as a diagnostic biomarker but could be taken to be a feature "risk factor" in view of the odds ratio of almost 2 recorded on the population having the highest CSF A $\beta 40$ concentration. The increased A $\beta 40$ might be a consequence of a reduced clearance of amyloid peptides in sporadic cases and/or a higher production-lower degradation. This matches the fact that in autosomal dominant forms of AD linked to APP or presenilin mutations [3, 5] and in Down syndrome [4], an overproduction of amyloid peptides is thought to trigger the AD process, along with all its consequences, including tau protein hyper-phosphorylation, in particular.

In this context, baseline $\mathrm{A} \beta 40$ concentration could indicate subjects with risk of early $\mathrm{AD}$ development. The positive correlation found to exist in the present study between $\mathrm{A} \beta 40$ and p-tau (181) in AD is an additional argument supporting this pathophysiological model. Tau has many phosphorylated isoforms [62, 63], some of them believed to be more specific for AD than p-tau (181), highlighting the pathophysiological role and therapeutic interest of kinases like PKA, CAMkII, or Cdk5. This isoform is however one of the best indicators of AD pathology in the CSF where it begins to increase as two decades before the development of aggregated tau pathology [44]. In this work, we had to rely only on the correlation with p-tau (181) because it is the only isoform with in vitro diagnostic (IVD) certification and has been measured in large clinical cohorts. The fact that this correlation was also present in a control population including a subgroup of welldefined FTD [52] and multiple sclerosis patients [51] raises many questions, however. It is tempting to take 
this relationship to confirm that $A \beta$ peptides may induce the phosphorylation of tau, as observed both in vitro and in vivo [64].

\section{Limitation}

Since the present study was based on a cross-sectional design, and without neuropathological confirmation, further studies involving a longitudinal design are now required to confirm the idea that high baseline CSF levels of $A \beta$ peptides may have prejudicial effects, leading to $\mathrm{AD}$.

\section{Conclusions}

In conclusion, our results indicate that an increase in the baseline level of amyloid peptides, which are associated with an increase in p-tau (181), could be a biological characteristic of AD. Further studies will be needed to establish a causal link between increased baseline levels of $A \beta 40$ and the development of the disease.

\section{Supplementary information}

Supplementary information accompanies this paper at https://doi.org/10. 1186/s13195-020-00696-1.

Additional file 1 : SupTable 1. Number of patients/samples in each clinical group and $A \beta 40$ detection method used.

Additional file $\mathbf{2}$ : Sup-Table 2. Percentage of AD/ADNI(+) patients in the four classes based on the A 440 percentile values recorded in the various cohorts; p25: 25th percentile, p25-50: 25th-50th percentile; p5075: 50th-75th percentile; p75: 75th.

Additional file 3 : Sup-Figure 1. CSF $A \beta 42$ and $A \beta 40$ in Non- $A D$ and $A D$ populations with regards to ApoE status Mean-centered values of CSF A 442 (panels A-C) and AB40 (panels D-F) in total (all), NAD and AD population with regards to ApoE4 allele presence (+) or absence (-). Panels G-I illustrate the difference in $A \beta 40$ between $A D$ and NAD in the population with the ApoE status determine, in the total (all), ApoE4 allele presence $(+)$ or absence (-).

Additional file 4 : Sup-Figure 2. $R O C$ curves of $A \beta 40, A \beta 42$ and $A \beta 42 /$ 40 ROC curves of $A \beta 40, A \beta 42$ and $A \beta 42 / 40$ for the detection of $A D$ in different cohorts. AUC of $A \beta 40$ had lower values than the other biomarkers (Mtp-1 0.686 (0.638 to 0.731); Mtp-2 0.751 (0.711 to 0.788); Paris 0,679 (0.641 to 0.716); SPIN-Barcelona $0.730(0.667$ to 0,787$)$ ).

Additional file 5 : Sup-Figure 3. A $A 40$ in different clinical groups. The Montpellier, Paris and SPIN-Barcelona cohorts displayed a large range of pathological samples from patients with Alzheimer's disease (AD), cerebral amyloid angiopathy (CAA), frontotemporal degeneration (FTD), mild cognitive impairment $(\mathrm{MCI})$, normal pressure hydrocephalus (NPH), vascular dementia (VASC), other neurological diseases (amytrophic lateral sclerosis, Parkinson's disease, Lewy Body dementia..) (Other) and control (subjective cognitive impairment). Box plots with median and 25th/75th percentile of CSF A 40 values are plotted for the four cohorts (panels AD). Note that the distribution of the diagnoses differ in the cohorts (see Methods). Non parametric Mann-Whitney U test were performed between the $\mathrm{AD}$ and the other clinical groups. $P$ values below statistical significance are in red.

\section{Abbreviations}

AD: Alzheimer's disease; ADNI: Alzheimer's Disease Neuroimaging Initiative; APP: Amyloid precursor protein; AUC: Area under the curve; AB: Amyloid $B$; CAA: Cerebral amyloid angiopathy; Cl: Confidence interval; CLIA: Chemiluminescence immunoassay; CMRR: Centre Mémoire de Ressources et de Recherche; CSF: Cerebrospinal fluid; FTD: Frontotemporal degeneration; MCI: Mild cognitive impairment; MMSE: Mini-Mental State Examination; MRI: Magnetic resonance brain imaging; NAD: Non-Alzheimer's disease; NFT: Neurofibrillary tangle; NPH: Normal pressure hydrocephalus; PET: Positron emission tomography; PiB: Pittsburgh compound B; PLM: ParisLille-Montpellier; QC: Quality control; ROC: Receiver operating characteristic; SD: Standard deviation; SCl: Subjective cognitive impairment

\section{Acknowledgements}

Not applicable.

\section{Authors' contributions}

Lehmann, Dumurgier, Lleo, and Gabelle had full access to all the data in the study and take responsibility for their presentation and the accuracy of the analysis. Concept and design: Dumurgier, Gabelle, and Lehmann. Acquisition of the data (biological and clinical): Ayrignac, Marelli, Alcolea, Fortea, Thouvenot, Delaby, Hirtz, Vialaret, Ginestet, Bouaziz-Amar, Laplanche, Labauge, and Paquet. Drafting of the manuscript: Dumurgier, Gabelle, and Lehmann. Administrative, technical, and material support: Hirtz and Delaby. The authors read and approved the final manuscript

\section{Funding}

This work was supported by "France Alzheimer" and via the French National Alzheimer effort ("Le Plan Alzheimer") and by the JPND project BiomarkAPD. Data collection and sharing for this project was also funded by the Alzheimer's Disease Neuroimaging Initiative (ADNI) (National Institutes of Health Grant U01 AG024904) and DOD ADNI (Department of Defense award number W81XWH-12-2-0012). ADNI is funded by the National Institute on Aging, the National Institute of Biomedical Imaging and Bioengineering, and through generous contributions from the following: AbbVie, Alzheimer's Association; Alzheimer's Drug Discovery Foundation; Araclon Biotech; BioClinica, Inc:; Biogen; Bristol-Myers Squibb Company; CereSpir, Inc.; Cogstate; Eisai Inc.; Elan Pharmaceuticals, Inc.; Eli Lilly and Company; Eurolmmun; F. Hoffmann-La Roche Ltd. and its affiliated company Genentech, Inc.; Fujirebio; GE Healthcare; IXICO Ltd.; Janssen Alzheimer Immunotherapy Research \& Development, LLC; Johnson \& Johnson Pharmaceutical Research \& Development LLC; Lumosity; Lundbeck; Merck \& Co., Inc.; Meso Scale Diagnostics, LLC; NeuroRx Research; Neurotrack Technologies; Novartis Pharmaceuticals Corporation; Pfizer Inc;; Piramal Imaging; Servier; Takeda Pharmaceutical Company; and Transition Therapeutics. The Canadian Institutes of Health Research is providing funds to support ADNI clinical sites in Canada. Private sector contributions are facilitated by the Foundation for the National Institutes of Health (www.fnih.org). The grantee organization is the Northern California Institute for Research and Education, and the study is coordinated by the Alzheimer's Therapeutic Research Institute at the University of Southern California. ADNI data are disseminated by the Laboratory for Neuro Imaging at the University of Southern California.

\section{Availability of data and materials}

The datasets used for the analyses are available from the corresponding author on reasonable request.

Ethics approval and consent to participate

All the patients at each clinical center gave their written informed consent to participating in clinical research on CSF biomarkers, which was approved by the respective Ethics Committees. The committee responsible in Montpellier was the regional Ethics Committee of the Montpellier University Hospital and Montpellier CSF-Neurobank \#DC-2008-417 at the certified NFS 96-900 CHU resource center BB-0033-00031, www.biobanques.eu. Authorization to handle personal data was granted by the French Data Protection Authority (CNIL) under the number 1709743 vo.

\section{Consent for publication}

Not applicable.

\section{Competing interests}

The authors report no conflict of interest to disclose.

\section{Author details}

'Univ Montpellier, CHU Montpellier (LBPC-PPC), INSERM (IRMB, INM), Montpellier, France. ${ }^{2}$ Centre de Neurologie Cognitive et Service de Biochimie et de Biologie Moléculaire, Groupe Hospitalier Lariboisière Fernand-Widal, INSERMU942, Université Paris Diderot, Paris, France. ${ }^{3} \mathrm{CHU}$ de Montpellier, 
Département de Neurologie, INSERM, Univ Montpellier, Montpellier, France. ${ }^{4}$ Sant Pau Memory Unit, Department of Neurology, Universitat Autònoma de Barcelona, Barcelona, Spain. ${ }^{5} \mathrm{CHU}$ de Nîmes, Département de Neurologie, INSERM, Univ Montpellier, Montpellier, France. ${ }^{6}$ Univ Montpellier, INSERM, CHU Montpellier (CMRR), Montpellier, France.

Received: 3 June 2020 Accepted: 23 September 2020

Published online: 02 October 2020

\section{References}

1. Blennow K, de Leon MJ, Zetterberg H. Alzheimer's disease. Lancet. 2006;368: 387-403.

2. Bloom GS. Amyloid-beta and tau: the trigger and bullet in Alzheimer disease pathogenesis. JAMA Neurol. 2014;71:505-8.

3. Bertram L, Lill CM, Tanzi RE. The genetics of Alzheimer disease: back to the future. Neuron. 2010;68:270-81.

4. Wiseman FK, Al-Janabi T, Hardy J, Karmiloff-Smith A, Nizetic D, Tybulewicz $\mathrm{VL}$, et al. A genetic cause of Alzheimer disease: mechanistic insights from Down syndrome. Nat Rev Neurosci. 2015;16:564-74.

5. Rovelet-Lecrux A, Hannequin D, Raux G, Le Meur N, Laquerriere A Vital A, et al. APP locus duplication causes autosomal dominant earlyonset Alzheimer disease with cerebral amyloid angiopathy. Nat Genet. 2006;38:24-6

6. Strittmatter WJ, Weisgraber KH, Huang DY, Dong LM, Salvesen GS, PericakVance $M$, et al. Binding of human apolipoprotein $E$ to synthetic amyloid beta peptide: isoform-specific effects and implications for late-onset Alzheimer disease. Proc Natl Acad Sci U S A. 1993;90:8098-102.

7. Brothers HM, Gosztyla ML, Robinson SR. The physiological roles of amyloidbeta peptide hint at new ways to treat Alzheimer's disease. Front Aging Neurosci. 2018;10:118.

8. Pernegre C, Duquette A, Leclerc N. Tau secretion: good and bad for neurons. Front Neurosci. 2019;13:649.

9. Andreasen N, Blennow K. CSF biomarkers for mild cognitive impairment and early Alzheimer's disease. Clin Neurol Neurosurg. 2005;107:165-73.

10. Blennow K, Hampel H. CSF markers for incipient Alzheimer's disease. Lancet Neurol. 2003;2:605-13.

11. McKhann GM, Knopman DS, Chertkow H, Hyman BT, Jack CR Jr, Kawas CH, et al. The diagnosis of dementia due to Alzheimer's disease: recommendations from the National Institute on Aging-Alzheimer's Association workgroups on diagnostic guidelines for Alzheimer's disease. Alzheimers Dement. 2011:7:263-9.

12. Dubois B, Feldman HH, Jacova C, Hampel H, Molinuevo JL, Blennow K, et al. Advancing research diagnostic criteria for Alzheimer's disease: the IWG-2 criteria. Lancet Neurol. 2014;13:614-29.

13. Gabelle A, Dumurgier J, Vercruysse $\mathrm{O}$, Paquet C, Bombois S, Laplanche JL, et al. Impact of the 2008-2012 French Alzheimer Plan on the use of cerebrospinal fluid biomarkers in research memory center: the PLM Study. J Alzheimers Dis. 2013:34:297-305.

14. Blennow K, Dubois B, Fagan AM, Lewczuk P, de Leon MJ, Hampel H. Clinical utility of cerebrospinal fluid biomarkers in the diagnosis of early Alzheimer's disease. Alzheimers Dement. 2015;11:58-69.

15. Lewczuk P, Kornhuber J, Toledo JB, Trojanowski JQ, Knapik-Czajka M, Peters $\mathrm{O}$, et al. Validation of the Erlangen score algorithm for the prediction of the development of dementia due to Alzheimer's disease in pre-dementia subjects. J Alzheimers Dis. 2015;48:433-41.

16. Nordberg A. PET imaging of amyloid in Alzheimer's disease. Lancet Neurol. 2004;3:519-27.

17. Villemagne $\mathrm{VL}$, Fodero-Tavoletti MT, Masters $\mathrm{CL}$, Rowe CC. Tau imaging: early progress and future directions. Lancet Neurol. 2015;14:114-24.

18. Jack CR Jr, Wiste HJ, Vemuri P, Weigand SD, Senjem ML, Zeng G, et al. Brain beta-amyloid measures and magnetic resonance imaging atrophy both predict time-to-progression from mild cognitive impairment to Alzheimer's disease. Brain. 2010;133:3336-48.

19. Gabelle A, Roche S, Geny C, Bennys K, Labauge P, Tholance Y, et al. Correlations between soluble alpha/beta forms of amyloid precursor protein and Abeta38, 40, and 42 in human cerebrospinal fluid. Brain Res. 2010;1357: $175-83$

20. Dorey A, Perret-Liaudet A, Tholance Y, Fourier A, Quadrio I. Cerebrospinal fluid Abeta40 improves the interpretation of Abeta42 concentration for diagnosing Alzheimer's disease. Front Neurol. 2015;6:247.
21. Lewczuk P, Esselmann H, Otto M, Maler JM, Henkel AW, Henkel MK, et al. Neurochemical diagnosis of Alzheimer's dementia by CSF A $342, A \beta 42 / A \beta 40$ ratio and total tau. Neurobiol Aging. 2004;25:273-81.

22. Lehmann S, Delaby C, Boursier G, Catteau C, Ginestet N, Tiers L, et al. Relevance of Abeta42/40 ratio for detection of Alzheimer disease pathology in clinical routine: the PLMR scale. Front Aging Neurosci. 2018;10:138.

23. Gervaise-Henry C, Watfa G, Albuisson E, Kolodziej A, Dousset B, Olivier JL, et al. Cerebrospinal fluid Abeta42/Abeta40 as a means to limiting tube- and storage-dependent pre-analytical variability in clinical setting. J Alzheimers Dis. 2017:57:437-45.

24. Lewczuk P, Lelental N, Spitzer P, Maler JM, Kornhuber J. Amyloid-beta 42/40 cerebrospinal fluid concentration ratio in the diagnostics of Alzheimer's disease: validation of two novel assays. J Alzheimers Dis. 2015;43:183-91.

25. Willemse E, van Uffelen K, Brix B, Engelborghs S, Vanderstichele H, Teunissen C. How to handle adsorption of cerebrospinal fluid amyloid beta (1-42) in laboratory practice? Identifying problematic handlings and resolving the issue by use of the Abeta42/Abeta40 ratio. Alzheimers Dement. 2017;13: 885-92.

26. Hansson O, Lehmann S, Otto M, Zetterberg $H$, Lewczuk P. Advantages and disadvantages of the use of the CSF amyloid beta (Abeta) $42 / 40$ ratio in the diagnosis of Alzheimer's disease. Alzheimers Res Ther. 2019:11:34.

27. Dumurgier J, Schraen S, Gabelle A, Vercruysse O, Bombois S, Laplanche JL, et al. Cerebrospinal fluid amyloid-beta $42 / 40$ ratio in clinical setting of memory centers: a multicentric study. Alzheimers Res Ther. 2015;7:30.

28. Wiltfang J, Esselmann $\mathrm{H}$, Bibl M, Hull M, Hampel $\mathrm{H}$, Kessler $\mathrm{H}$, et al. Amyloid beta peptide ratio $42 / 40$ but not A beta 42 correlates with phospho-Tau in patients with low- and high-CSF A beta 40 load. J Neurochem. 2007;101: 1053-9.

29. Bibl M, Mollenhauer B, Lewczuk $P$, Esselmann $H$, Wolf $S$, Otto $M$, et al. Cerebrospinal fluid tau, p-tau 181 and amyloid-beta38/40/42 in frontotemporal dementias and primary progressive aphasias. Dement Geriatr Cogn Disord. 2011;31:37-44.

30. Gabelle A, Roche S, Geny C, Bennys K, Labauge P, Tholance Y, et al. Decreased sAbetaPPbeta, Abeta38, and Abeta40 cerebrospinal fluid levels in frontotemporal dementia. J Alzheimers Dis. 2011;26:553-63.

31. Renard D, Castelnovo G, Wacongne A, Le Floch A, Thouvenot E, Mas J, et al. Interest of CSF biomarker analysis in possible cerebral amyloid angiopathy cases defined by the modified Boston criteria. J Neurol. 2012;259:2429-33.

32. Gisslen M, Krut J, Andreasson U, Blennow K, Cinque P, Brew BJ, et al. Amyloid and tau cerebrospinal fluid biomarkers in HIV infection. BMC Neurol. 2009;9:63.

33. Pietroboni AM, Caprioli M, Carandini T, Scarioni M, Ghezzi L, Arighi A, et al. CSF beta-amyloid predicts prognosis in patients with multiple sclerosis. Mult Scler. 2019;25:1223-31.

34. Chen Z, Liu C, Zhang J, Relkin N, Xing Y, Li Y. Cerebrospinal fluid Abeta42, ttau, and p-tau levels in the differential diagnosis of idiopathic normalpressure hydrocephalus: a systematic review and meta-analysis. Fluids Barriers CNS. 2017;14:13.

35. Olsson B, Lautner R, Andreasson U, Öhrfelt A, Portelius E, Bjerke M, et al. CSF and blood biomarkers for the diagnosis of Alzheimer's disease: a systematic review and meta-analysis. Lancet Neurol. 2016;15:673-84.

36. Alcolea D, Martinez-Lage P, Sanchez-Juan P, Olazaran J, Antunez C, Izagirre A, et al. Amyloid precursor protein metabolism and inflammation markers in preclinical Alzheimer disease. Neurology. 2015;85:626-33.

37. Sala I, IIlan-Gala I, Alcolea D, Sanchez-Saudinos MB, Salgado SA, MorenasRodriguez $\mathrm{E}$, et al. Diagnostic and prognostic value of the combination of two measures of verbal memory in mild cognitive impairment due to Alzheimer's disease. J Alzheimers Dis. 2017;58:909-18.

38. McKhann GM, Albert MS, Grossman M, Miller B, Dickson D, Trojanowski JQ. Clinical and pathological diagnosis of frontotemporal dementia: report of the Work Group on Frontotemporal Dementia and Pick's Disease. Arch Neurol. 2001;58:1803-9.

39. McKeith IG. Consensus guidelines for the clinical and pathologic diagnosis of dementia with Lewy bodies (DLB): report of the Consortium on DLB International Workshop. J Alzheimers Dis. 2006;9:417-23.

40. Boeve BF, Maraganore DM, Parisi JE, Ahlskog JE, Graff-Radford N, Caselli RJ, et al. Pathologic heterogeneity in clinically diagnosed corticobasal degeneration. Neurology. 1999;53:795-800.

41. Lehmann S, Schraen S, Paquet C, Bombois S, Delaby C, Dorey A, et al. A diagnostic scale for Alzheimer's disease based on cerebrospinal fluid biomarker profiles. Alzheimers Res Ther. 2014;6:38. 
42. Dumurgier J, Vercruysse O, Paquet C, Bombois S, Chaulet C, Laplanche JL, et al. Intersite variability of CSF Alzheimer's disease biomarkers in clinical setting. Alzheimers Dement. 2013;9:406-13.

43. del Campo M, Mollenhauer B, Bertolotto A, Engelborghs S, Hampel H, Simonsen $\mathrm{AH}$, et al. Recommendations to standardize preanalytical confounding factors in Alzheimer's and Parkinson's disease cerebrospinal fluid biomarkers: an update. Biomark Med. 2012;6:419-30.

44. Barthelemy NR, Li Y, Joseph-Mathurin N, Gordon BA, Hassenstab J, Benzinger TLS, et al. A soluble phosphorylated tau signature links tau, amyloid and the evolution of stages of dominantly inherited Alzheimer's disease. Nat Med. 2020;26:398-407.

45. Hansson O, Seibyl J, Stomrud E, Zetterberg H, Trojanowski JQ, Bittner T, et al. CSF biomarkers of Alzheimer's disease concord with amyloid-beta PET and predict clinical progression: a study of fully automated immunoassays in BioFINDER and ADNI cohorts. Alzheimers Dement. 2018;14:1470-81.

46. Spellman DS, Wildsmith KR, Honigberg LA, Tuefferd M, Baker D, Raghavan N, et al. Development and evaluation of a multiplexed mass spectrometry based assay for measuring candidate peptide biomarkers in Alzheimer's Disease Neuroimaging Initiative (ADNI) CSF. Proteomics Clin Appl. 2015;9:715-31.

47. Perret-Liaudet A, Pelpel M, Tholance $Y$, Dumont B, Vanderstichele H, Zorzi $W$, et al. Risk of Alzheimer's disease biological misdiagnosis linked to cerebrospinal collection tubes. J Alzheimers Dis. 2012;31:13-20.

48. Renard D, Wacongne A, Ayrignac X, Charif M, Fourcade G, Azakri S, et al. Cerebrospinal fluid Alzheimer's disease biomarkers in cerebral amyloid angiopathy-related inflammation. J Alzheimers Dis. 2015;50:759-64.

49. Peskind ER, Li G, Shofer J, Quinn JF, Kaye JA, Clark CM, et al. Age and apolipoprotein $E^{*} 4$ allele effects on cerebrospinal fluid beta-amyloid 42 in adults with normal cognition. Arch Neurol. 2006;63:936-9.

50. Sunderland T, Mirza N, Putnam KT, Linker G, Bhupali D, Durham R, et al. Cerebrospinal fluid beta-amyloid1-42 and tau in control subjects at risk for Alzheimer's disease: the effect of APOE epsilon4 allele. Biol Psychiatry. 2004; 56:670-6.

51. Vukusic S, Casey R, Rollot F, Brochet B, Pelletier J, Laplaud DA, et al. Observatoire Francais de la Sclerose en Plaques (OFSEP): a unique multimodal nationwide MS registry in France. Mult Scler. 2020;26:118-22.

52. Marelli C, Gutierrez LA, Menjot de Champfleur N, Charroud C, De Verbizier D, Touchon J, et al. Late-onset behavioral variant of frontotemporal lobar degeneration versus Alzheimer's disease: interest of cerebrospinal fluid biomarker ratios. Alzheimers Dement (Amst). 2015;1:371-9.

53. Janelidze S, Zetterberg H, Mattsson N, Palmqvist S, Vanderstichele $H$, Lindberg $\mathrm{O}$, et al. CSF Abeta42/Abeta40 and Abeta42/Abeta38 ratios: better diagnostic markers of Alzheimer disease. Ann Clin Transl Neurol. 2016:3:154-65.

54. Welge V, Fiege O, Lewczuk P, Mollenhauer B, Esselmann H, Klafki HW, et al, Combined CSF tau, p-tau181 and amyloid-beta 38/40/42 for diagnosing Alzheimer's disease. J Neural Transm. 2009;116:203-12.

55. Sundelof J, Sundstrom J, Hansson O, Eriksdotter-Jonhagen M, Giedraitis V, Larsson A, et al. Higher cathepsin B levels in plasma in Alzheimer's disease compared to healthy controls. J Alzheimers Dis. 2010;22:1223-30.

56. Lewczuk P, Matzen A, Blennow K, Parnetti L, Molinuevo JL, Eusebi P, et al. Cerebrospinal fluid Abeta42/40 corresponds better than Abeta42 to amyloid PET in Alzheimer's disease. J Alzheimers Dis. 2017:55:813-22.

57. Tijms BM, Vermunt $L$, Zwan MD, van Harten $A C$, van der Flier WM

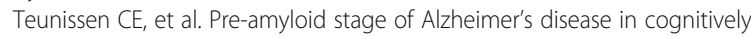
normal individuals. Ann Clin Transl Neurol. 2018:5:1037-47.

58. Janelidze S, Pannee J, Mikulskis A, Chiao P, Zetterberg H, Blennow K, et al. Concordance between different amyloid immunoassays and visual amyloid positron emission tomographic assessment. JAMA Neurol. 2017;74:1492-501.

59. Nakamura A, Kaneko N, Villemagne VL, Kato T, Doecke J, Dore V, et al. High performance plasma amyloid-beta biomarkers for Alzheimer's disease. Nature. 2018;554:249-54.

60. Hanon O, Vidal JS, Lehmann S, Bombois S, Allinquant B, Treluyer JM, et al. Plasma amyloid levels within the Alzheimer's process and correlations with central biomarkers. Alzheimers Dement. 2018.

61. Gabelle A, Schraen S, Gutierrez LA, Pays C, Rouaud O, Buee L, et al. Plasma beta-amyloid 40 levels are positively associated with mortality risks in the elderly. Alzheimers Dement. 2018;14:858-68.

62. Simic G, Babic Leko M, Wray S, Harrington C, Delalle I, Jovanov-Milosevic N, et al. Tau protein hyperphosphorylation and aggregation in Alzheimer's disease and other tauopathies, and possible neuroprotective strategies. Biomolecules. 2016;6:6.
63. Martin L, Latypova X, Terro F. Post-translational modifications of tau protein: implications for Alzheimer's disease. Neurochem Int. 2011;58:458-71.

64. Selenica ML, Brownlow M, Jimenez JP, Lee DC, Pena G, Dickey CA, et al. Amyloid oligomers exacerbate tau pathology in a mouse model of tauopathy. Neurodegener Dis. 2013;11:165-81.

\section{Publisher's Note}

Springer Nature remains neutral with regard to jurisdictional claims in published maps and institutional affiliations.
Ready to submit your research? Choose BMC and benefit from:

- fast, convenient online submission

- thorough peer review by experienced researchers in your field

- rapid publication on acceptance

- support for research data, including large and complex data types

- gold Open Access which fosters wider collaboration and increased citations

- maximum visibility for your research: over $100 \mathrm{M}$ website views per year

At BMC, research is always in progress.

Learn more biomedcentral.com/submissions 